\title{
HARDY-BOHR POSITIVITY
}

\author{
J. DEFRANZA AND K. ZELLER
}

(Communicated by Christopher D. Sogge)

\begin{abstract}
We consider two general principles that lead to Hardy-Bohr positivity. These are applied to give a simple proof that the Cesàro methods of positive order have Hardy-Bohr positivity.
\end{abstract}

\section{INTRODUCTION}

For matrix methods of summability $A$, where $A$ is a regular matrix triangle, determining the summability factors has attracted the attention of many authors. In the theory of sequence spaces the notion of a sum space and the so-called Hardy-Bohr positivity play important roles. We consider two general principles that lead to Hardy-Bohr positivity. In section 3 we show that any triangle with a diapositive inverse has Hardy-Bohr positivity and apply this to show that the Cesàro methods $(C, \alpha)$, for $0 \leq \alpha \leq 1$, have this property. In section 4 we consider convolution triangles and show that the product of two convolution triangles with Hardy-Bohr positivity also has Hardy-Bohr positivity. This result together with the result of section 3 gives Hardy-Bohr positivity for the Cesàro methods of all orders $\alpha \geq 0$. The proof for the Cesàro methods is significantly easier than any previously given. In section 5 we conclude with several comments on Nörlund methods, which are not Cesàro-like and have Hardy-Bohr positivity.

\section{NOTATION AND TERMINOLOGY}

Throughout we use notation and results given by Wilansky [20] and by ZellerBeekmann [21]. Let $\omega$ denote the space of all sequences, $m$ the space of bounded sequences, $c$ the convergent sequences, $c_{0}$ sequences that converge to $0, c s=\left\{x: \sum_{n} x_{n}\right.$ is convergent $\}, l_{1}=\left\{x: \sum_{n}\left|x_{n}\right|<\infty\right\}$, and $\varphi$ all finitely nonzero sequences. If $A=\left(a_{n k}\right)$ is an infinite matrix, the matrix method $A$ defines a sequence-to-sequence transformation, mapping a sequence $s$ (real or complex terms) to $t$

$$
t_{n}=(A x)_{n}=\sum_{k=0}^{\infty} a_{n k} s_{k}, \quad n=0,1,2, \ldots
$$

Received by the editors April 4, 1994 and, in revised form, June 9, 1994.

1991 Mathematics Subject Classification. Primary 40C05, 40G99, 40H05, 46A45. 
The convergence domain $c_{A}$ for the matrix method $A$ consists of those sequences $s$ for which $t=A s$ exists and belongs to $c$. The $A$-limit is defined for $s \in c_{A}$ by $A-\lim s_{k}=\lim t_{n}$. The method $A$ is conservative provided $c \subset c_{A}$ and strictly stronger than convergence if, moreover, $c \neq c_{A}$. A conservative method is regular provided $A-\lim s_{k}=\lim s_{k}$ for all $s \in c$. The matrix $A$ is a triangle provided $a_{n k}=0$ for $k>n$ and $a_{n n} \neq 0$ for all $n$. Let $\Sigma$ denote the triangle of ones, so that $A \Sigma$ is the series-to-sequence method associated with $A$.

If $E$ is an $F K$-space containing $\varphi$, the multipliers on $E$ are defined as $M(E)=\{x \in \omega: x y \in E \forall x \in E\}$, where $x y$ denotes the coordinatewise product. The $f$-dual of $E$ is $E^{f}=\left\{\left(f\left(e_{i}\right)\right)_{i=1}^{\infty}: f \in E^{\prime}\right\}$ where $E^{\prime}$ is the topological dual of $E$ and $e_{i}$ denotes the $i$ th coordinate sequence. Then $E$ is said to be a sum space provided $E^{f}=M(E)$. The notion of a sum space was defined and studied by Ruckle in [16], [17], [18], and [19]. For example, let $B$ be a $c$-reversible, row-finite, $\mathrm{sp}_{1}$ matrix (i.e., column limits are all one) such that $l_{1} \subseteq c_{B}$ or $c_{B}$ has $A D$ (i.e., $\varphi$ is dense). Then $c_{B}$ is a sum space if and only if $c_{B}$ has $B$-sectional boundedness if and only if $c_{B}$ has $B$-sectional convergence [16], [8]. In particular, if $A$ is a regular triangle, then $B=A \Sigma$ is a $c$-reversible, row-finite, $\mathrm{sp}_{1}$ matrix such that $l_{1} \subseteq c_{B}$. In [22], Zeller showed that if $B=(C, \alpha) \Sigma, \alpha \geq 0$, then $c_{B}$ has $B$-sectional convergence and hence the series-to-sequence Cesàro methods of order $\alpha \geq 0$ are sum spaces. If a sequence $\beta$ has the property that $\sum_{n} \beta_{n} s_{n}$ is summable $B$ whenever $\sum_{n} s_{n}$ is summable $A$ we say $\beta$ is a summability factor of type $(A, B)$ and write $\beta \in(A, B)$. For a triangle $A=\left(a_{n k}\right)$ (sequence-to-sequence method) let $A \Sigma=$ $\left(\bar{a}_{n k}\right)$ be the corresponding series-to-sequence method where $\bar{a}_{n k}=\sum_{j=k}^{n} a_{n j}$ if $k \leq n$ and $\bar{a}_{n k}=0$ if $k>n$. An ordered pair of triangles $[A, B]$ belongs to the class $\mathscr{L}$, or satisfies the Hardy-Bohr criteria, if the conditions

$$
\begin{aligned}
& \beta_{n}=d+\sum_{j=n}^{\infty} \bar{a}_{j n} \gamma_{j}, \\
& \beta_{n}=O\left(a_{n n} / b_{n n}\right)
\end{aligned}
$$

where $d$ is a constant and $\gamma=\left(\gamma_{j}\right) \in l_{1}(d$ and $\gamma$ depend on $\beta)$ are necessary and sufficient for $\beta \in(A, B)$. It follows from [15], [7], [13], and [14] that $[(C, \alpha),(C, \beta)] \in \mathscr{L}, \forall \alpha, \beta \geq 0$. Many authors have considered the problem of identifying pairs of methods in the class $\mathscr{L}$. In [9] the observation is made that for a regular triangle $A,[A, A] \in \mathscr{L}$ if and only if $c_{A \Sigma}$ is a sum space. Moreover, the regular triangular methods $A$ for which $[A, A] \in \mathscr{L}$ are precisely those methods for which the summability factors represent the continuous linear functionals on $c_{A \Sigma}$. A regular triangle $A$ is said to have Hardy-Bohr positivity, or HB-positivity, provided

$$
\sum_{l=j}^{k}\left(a_{i j}^{-1}-a_{i-1, j}^{-1}\right) \bar{a}_{n l} \bar{a}_{k l} \geq 0, \quad \forall 0 \leq j \leq k \leq n,
$$

where $A^{-1}=\left(a_{n k}^{-1}\right)$. If $A$ has HB-positivity, then the series-to-sequence convergence domain $c_{A \Sigma}$ is a sum space [9]. Let $\Sigma^{\alpha}, \alpha \geq 0$, be the convolution method generated by $p(x)=1 /(1-x)^{\alpha}$. In [12] it is shown that $\Sigma^{\alpha}$ has HBpositivity, and from this one easily argues the HB-positivity of $(C, \alpha)$. In this 
article, we establish the HB-positivity of $\Sigma^{\alpha}$ for $0 \leq \alpha \leq 1$ using Theorem 1, whereas, the HB-positivity of $\Sigma^{\alpha}$ for $\alpha>1$ then follows from Theorem 2. See [12], [1], [10], [2], [3], [4], [5], [6], and [21].

For a triangle $A$ and $B=A \Sigma$ we let $D_{r}$ denote the diagonal matrix with the $r$ th row of $B$ on the diagonal. That is $d_{k k}=b_{r k}$ for all $k \geq 0$. Then $A$ having HB-positivity is equivalent to

$$
B D_{r} B^{-1} \geq 0, \quad \forall r .
$$

In the next two sections we give two general principles leading to HB-positivity together covering the Cesàro methods and yielding a simple proof.

\section{Diapositive inverse}

A triangle $A=\left(a_{n k}\right)$ is said to be diapositive provided $a_{n n}>0$ for all $n \geq 0$ and $a_{n k} \leq 0$ for $n \neq k$. See [21] and [23].

Theorem 1. Let $A$ be a regular triangle and $B=A \Sigma$. If $A^{-1}$ is diapositive, then $A$ has HB-positivity.

Proof. First we observe that $A \geq 0$ since $A^{-1}$ is diapositive (use geometric series or recursion). Hence in each row of $B$ the elements decrease to 0 (and are eventually all equal to 0 ). Next we consider $B D_{r} B^{-1} A=B D_{r} \Sigma^{-1}$. Then the elements of row $m$ of this matrix are given by

$$
b_{m, 0} b_{r, 0}-b_{m, 1} b_{r, 1}, b_{m, 1} b_{r, 1}-b_{m, 2} b_{r, 2}, \ldots,
$$

and since $B \Sigma^{-1}=A$, the elements in row $m$ are given by

$$
a_{r, 0} b_{m, 0}+a_{m, 0} b_{r, 1}, a_{r, 1} b_{m, 1}+a_{m, 1} b_{r, 2}, \ldots
$$

The terms $a_{r, k} b_{m, k}$ for $r, k \geq 0$ constitute the matrix $A D_{m}$. Multiplying by $A^{-1}$ we are led to

$$
A D_{m} A^{-1} \geq 0
$$

where the inequality is true for the following reasons. The elements of row $r$ of this latter matrix are of the form

$$
a_{r, 0} a_{0,0}^{-1} b_{m, 0}+\cdots+a_{r, r} a_{r, 0}^{-1} b_{m, r}, a_{r, 1} a_{1,1}^{-1} b_{m, 1}+\cdots+a_{r, r} a_{r, 1}^{-1} b_{m, r}, \ldots
$$

In $A A^{-1}=I \geq 0$, modified by the terms of $B$, the influence of the negative terms is diminished in comparison to the positive term when multiplied by the decreasing factors of $D_{m}$. Similarly, the terms $a_{m, k} b_{r, k+1}$ constitute the matrix $A$ times $D_{m}$ shifted and when multiplied on the right by $A^{-1}$ the nonnegativity follows as above.

As an application of Theorem 1, since $\Sigma^{\alpha}$ for $0 \leq \alpha \leq 1$ has a diapositive inverse, the methods have HB-positivity and hence we have a simple proof that the Cesàro methods of order $\alpha, 0 \leq \alpha \leq 1$, also have HB-positivity.

\section{Convolution triangles}

Given a suitable sequence $p=\left(p_{k}\right)$ the triangle $A=\left(a_{n k}\right)=\left(p_{n-k}\right), n, k \geq$ 0 , is called a convolution triangle (or Toeplitz matrix) [11]. The product of two convolution triangles is another convolution triangle generated by the Cauchy 
product of the two original generating sequences. For the convolution triangle $A$ define the function $p$ by

$$
p(x)=\sum_{k=0}^{\infty} p_{k} x^{k}
$$

Let

$$
P(x)=\frac{p(x)}{(1-x)}=\sum_{k=0}^{\infty} P_{k} x^{k},
$$

where $P_{k}=\sum_{k=0}^{n} p_{k}$. Such a convolution triangle $A$ has HB-positivity if and only if the coefficients of the power series

$$
\frac{P(x) P(y)}{P(x y)}
$$

are nonnegative. That is the series is absolutely monotone [1]. Let

$$
A(x)=\frac{1-x}{p(x)}
$$

Then HB-positivity follows immediately from

$$
\begin{aligned}
Q & =\frac{P(x) P(y)}{P(x y)}=\sum_{i, j, k=0}^{\infty} P_{j} P_{k} A_{i}(x y)^{i} x^{k} y^{j} \\
& =\sum_{i, j, k=0}^{\infty} P_{j} P_{k} A_{i} x^{i+k} y^{i+j}
\end{aligned}
$$

and letting $m=i+k, n=i+j$,

$$
Q=\sum_{m, n=0}^{\infty}\left\{\sum_{i=0}^{\min (m, n)} P_{m-i} P_{n-i}\left(a_{i}-a_{i-1}\right)\right\} x^{m} y^{n}
$$

where $a(x)=1 / p(x)$. This is precisely the condition for HB-positivity [12].

Theorem 2. If $S$ and $T$ are convolution triangles with HB-positivity, then $S T$ has $H B$-positivity.

Proof. If the functions for $S$ and $T$ are $p$ and $q$ respectively, then the function associated with $S T$ is simply $p q$. The criterion together with the commutivity of $P$ and $Q$ leads to the condition that

$$
\frac{P(x) P(y)}{P(x y)} \frac{Q(x) Q(y)}{Q(x y)}
$$

is absolutely monotone.

As an application of Theorem 2 we have $\Sigma^{\alpha}$ for $\alpha \geq 1$ has HB-positivity and hence as before the Cesàro methods of order $\alpha \geq 1$ have HB-positivity. This follows from Theorem 1 and Theorem 2 and transfinite induction.

\section{REMARKS}

The Nörlund methods provide additional interesting non-Cesàro-like triangles with HB-positivity. In [10], the Nörlund method $N_{p}$ generated by $p(x)=$ 
$(1+x) /(1-x)$ is shown to have HB-positivity, so that $c_{N_{p} \Sigma}$ is a sum space and, moreover, $\left[N_{p}, N_{p}\right] \in \mathscr{L}$ so that the summability factors are given by the classical conditions. As in the case of $\Sigma^{\alpha}$ and $(C, \alpha)$, if the convolution triangle generated by the function $p(x)$ has HB-positivity, then the Nörlund mean generated by $p(x)$ will also have HB-positivity. Arguing as in Theorem 2 , if a convolution triangle generated by $p(x)$ has HB-positivity, then the convolution triangle generated by $p(x) /(1-x)^{\alpha}, \alpha \geq 0$, will also have HB-positivity. In particular, the Nörlund methods generated by $(1+x) /(1-x)^{\alpha}, \alpha \geq 1$, have HB-positivity. In a similar manner, if $M$ and $N$ are positive integers with $N \geq 2 M-1$, then the Nörlund method generated by

$$
p(x)=(1+x)^{M} /(1-x)^{N}
$$

has HB-positivity. Computer analysis suggests this latter result is best possible.

\section{REFERENCES}

1. R. Askey, G. Gasper, and M. Ismail, A positive sum from summability theory, J. Approx. Theory 13 (1975), 413-420.

2. W. Beekmann and K. Zeller, Normvergleich bei abschnittskeschräten Limitierungverfahren, Math. Z. 126 (1972), 116-122.

3. __ Positive Operatoren in der Limitierung, Internat. Schriftenreihe Numer. Math, vol. 25, Birkhäuser Verlag, Basel and Stuttgart, 1974, pp. 559-564.

4. __ Positivity in summability, Internat. Schriftenreihe Numer. Math., vol. 71, Birkhäuser Verlag, Basel, 1984, pp. 111-117.

5. _ـ Positivity in absolute summability, Internat. Schriftenreihe Numer. Math., vol. 80, Birkhäuser Verlag, Basel and Boston, 1986, pp. 205-215.

6. __ Inequalities for summability factors, Internat. Schriftenreihe Numer. Math., vol. 103, Birkhäuser Verlag, Basel and Boston, 1992, pp. 185-192.

7. L. S. Bosanquet, Note on convergence and summability factors III, Proc. London. Math. Soc. (2) 50 (1949), 482-496.

8. M. Buntinas, On Toeplitz sections in sequence spaces, Math. Proc. Cambridge Philos. Soc. 78 (1975), 451-460.

9. J. DeFranza and D. J. Fleming, Sequence spaces and summability factors, Math. Z. 199 (1988), 99-108.

10. _ On the Bohr-Hardy criteria, Arch. Math. 51 (1988), 464-473.

11. G. H. Golub and C. F. Van Loan, Matrix computations, Hopkins Univ. Press, Oxford, 1983.

12. G. G. Lorentz and K. Zeller, Abschnittslimitierbarkeit und der Satz von Hardy-Bohr, Arch. Math. 15 (1964), 208-213.

13. W. Jurkat and A. Peyerimhoff, Summierbarkeits-Faktoren, Math. Ann. 58 (1953), 186-208.

14. G. E. Peterson, Summability factors, Proc. London Math. Soc. (3) 19 (1969), 341-356.

15. A. Peyerimhoff, Über Summierbarkeitsfaktoren und verwandte Fragen bei Cesàro-Verfahren, Acad. Serbe. Sci. Publ. Inst. Math. 8 (1955), 139-156.

16. W. Ruckle, An abstract concept of the sum of a numerical series, Canad. J. Math. 22 (1970), 863-874.

17. __ A universal topology for sequence spaces, Math. Ann. 236 (1978), 43-48.

18. _ Topologies on sequence spaces, Pacific J. Math. 42 (1972), 234-249.

19. __ Sequence spaces, Pitman, London, 1981.

20. A. Wilansky, Summability through functional analysis, North-Holland, Amsterdam, 1984. 
21. K. Zeller and W. Beekmann, Theorie der Limitierungsverfahren, Springer-Verlag, Berlin, Heidelberg, and New York, 1970.

22. K. Zeller, Approximation in Wirkfeldern von Summierungsverfahren, Arch. Math. 4 (1953), 425-431.

23. __ Abschnittsabschätzungen bei Matrixransformationen, Math. Z. 80 (1963), 355-357.

Department of Mathematics, St. Lawrence University, Canton, New York 13617

Mathematische Fakutät, Universitat Tubingen, Auf Der Morgenstelle 10, D-7400, TÜBINGEN, GERMANY 